\title{
A novel cardioprotective mechanism of exogenous nitric oxide: inhibition of Rho-associated kinase activity
}

\author{
Atsushi Tanaka and Koichi Node \\ Hypertension Research (2015) 38, 461-462; doi:10.1038/hr.2015.61; published online 23 April 2015
}

$\mathrm{O}$ rganic nitrates have been used widely as antianginal drugs for many years, and have an established role in the management of patients with anginal syndromes, including stable angina on effort and coronary spastic angina. Their mode of action is to provide exogenous nitric oxide (NO) that decreases the cardiac preload and afterload principally through relaxation of vascular smooth muscle cells (VSMCs) as a consequence of a NOmediated increase of cyclic 3'5'-guanosine monophosphate. In addition, they are known to dilate the coronary arteries and to inhibit platelet aggregation. It is common for physicians to administer organic nitrates to patients with anginal syndromes for lengthy periods, presumably on the assumption that they are both effective and safe in this setting. On the other hand, although exogenous NO administration is commonly used to regulate hemodynamics in the acute phase, the cardioprotective effects of long-term administration in patients with acute or old myocardial infarction have been controversial. It may be difficult to establish clinical benefits in routine clinical practice, when technical progress in coronary intervention and new cardio-protective drugs (for example, statins, antiplatelet agents, $\beta$-adrenergic antagonists and renin-angiotensin inhibitors) are widely employed. ${ }^{1}$ Accordingly, more basic research is needed to identify the cardioprotective benefits through mechanisms that are additional to vasodilation that might then provide a basis for the development of novel therapies.

One of the essential pathologies of coronary atherosclerosis is inflammatory change in

A Tanaka and K Node are at Department of Cardiovascular Medicine, Saga University, Saga, Japan

E-mail: tanakaa2@cc.saga-u.ac.jp systemic and local arteries, leading to endothelial dysfunction. Recent research has shown that activated Rho-associated coiledcoil forming kinases (ROCKs) are implicated in endothelial dysfunction and various other pathologies, including those of the cardiovascular system, and has focused attention on the therapeutic potential of modifying the regulation of the ROCK pathway., ${ }^{2,3}$

ROCKs are serine/threonine kinases of $160 \mathrm{kDa}$, and are one of the downstream targets of RhoA that have diverse cellular functions. ${ }^{4,5}$ They are composed of a kinase domain, followed by a coiled-coil-forming domain that contains a Rho-binding domain, and a cysteine-rich domain located within a pleckstrin-homology domain. There are two isoforms, ROCK1 and ROCK2, that share high homology (65\% in the total amino-acid sequence, $92 \%$ in the kinase domains). Despite these similarities, the two isoforms seem different in their sites of expression, cellular localization and functions. However, as isoform-specific ROCK inhibitors are not currently commercially available, the precise differences between ROCK1 and ROCK2 in their molecular mechanisms have not yet been elucidated.

Recent evidence has uncovered several biological functions and roles of ROCKs. These include the cellular migration, proliferation, fibrosis and contraction of VSMCs; endothelial cell dysfunction mediated by decreased NO bioavailability; migration of macrophages and their transformation into foam cells; and hypertrophy and deleterious remodeling of the myocardium. ${ }^{4,5}$ Of all these activities, the effects on the contraction of VSMCs have been the most intensively investigated in vitro and in vivo. In experimental systems, the pathologically activated Rho/ROCK pathway has been shown to phosphorylate the myosin binding subunit of the myosin light chain (MLC) phosphatase, creating an imbalance between MLC phosphatase and MLC kinase that in turn leads to a calcium ion-independent hypercontraction of VSMCs. ${ }^{6}$ These findings have raised the possibility that ROCKs are intimately involved in the development of cardiovascular diseases.

Endothelium-derived NO has critical roles in the relaxation of neighboring VSMCs and in the inhibition of vascular remodeling. In addition, it negatively regulates RhoA activation through phosphorylation of RhoA at Ser188. ${ }^{7}$ Chitaley and $\mathrm{Webb}^{8}$ reported that NO inhibited RhoA/ROCK activity in the rat aorta, suggesting that endogenous NO-mediated vasodilation might be caused in part by suppression of RhoA/ROCK signaling. In contrast, activated RhoA/ROCK signaling destabilizes endothelial NO synthase mRNA and limits the bioavailability of $\mathrm{NO}$, leading to hypercontraction of the VSMCs and pathological cellular remodeling. Taken together, a physiological link between endothelial cells and VSMCs is effected by $\mathrm{NO}$ and the RhoA/ROCK pathways. It seems likely that regulation of these pathways has the potential to provide a novel mode of drug action, and a promising therapeutic target in cardiovascular diseases.

During the past 20 years, many in vivo and in vitro studies using experimental models, including studies of humans, have demonstrated that the RhoA/ROCK pathway has an important place in the pathophysiology of various cardiovascular diseases, including ischemic heart disease, coronary spastic angina, hypertension, heart failure, pulmonary hypertension and metabolic syndrome. It has also been shown that selective ROCK inhibitors, namely Y-27632 and fasudil, have 
reduced ROCK activity and improved clinical symptoms. Interestingly, in patients with microvascular angina, the so-called cardiac syndrome $\mathrm{X}$, fasudil decreased both acetylcholine provocation-mediated lactic acid production and anginal symptoms. ${ }^{9}$ Given these results, inhibition of the ROCK pathway may have beneficial effects on the cardiovascular system beyond those provided by vasodilation.

In this issue, Maruhashi et al. ${ }^{10}$ report that exogenous $\mathrm{NO}$ in the form of isosorbide mononitrate (40 mg per day) reduced leukocyte ROCK activity in 15 patients with angina pectoris on effort in a single-center randomized prospective study. The same group has previously reported that exogenous NO given as sodium nitroprusside inhibited angiotension II-induced ROCK activities in VSMCs in both in vitro and in vivo experimental models, including in humans. ${ }^{11}$ Their present report is the first in vivo study to demonstrate that exogenous NO decreased ROCK activity in patients with angina pectoris on effort, pointing to the potential effectiveness of targeting the ROCK pathway in the management of anginal syndromes. Greater insight into NO-mediated ROCK inhibition (for example, functional and/or structural improvement of endothelial cells, VSMCs or myocardial cells) might elucidate the role of exogenous $\mathrm{NO}$ in the primary and/or secondary prevention of various types of cardiovascular disease.

The inhibitory effect of isosorbide nitrate in the study of Maruhashi et al. ${ }^{10}$ was evaluated after 4 weeks of treatment, and was continued for 12 weeks without significant changes in clinical measurements, including body mass index, blood pressure, lipid profiles and serum glucose values. As the ROCK activities in the control group, who received conventional treatment of angina pectoris, were not reduced, it is suggested that exogenous NO selectively lowers ROCK activity, although details of coadministered drugs were not provided. As nitrates are often administered in the long term, it will be necessary to demonstrate whether the inhibitory effect is maintained for longer periods. In addition, a solution to the problem of nitrate tolerance will be needed.
In this study, leukocyte ROCK activity in peripheral blood was evaluated by western blotting tests using blood samples. The same team has shown previously that leukocyte ROCK activity was comparable to vascular ROCK activity, measured by the conventional physiological method. ${ }^{12}$ Others have reported that enhanced leukocyte ROCK activity was significantly associated with the pathology in coronary spastic angina. ${ }^{13}$ Given these results, leukocyte ROCK activity might be a useful biomarker that reflects local and/or systemic vascular disease risk. Although the clinical value of assaying leukocyte ROCK activity might also extend to other clinical settings, as the current procedure is based on western blotting, it will be necessary to develop a more convenient method for routine practice.

The authors report that ROCK activity is regulated by conformational change, not by protein level. Indeed, although ROCK activity was clearly reduced by exogenous $\mathrm{NO}$ in patients with angina pectoris, ROCK1 and ROCK2 expression levels were not altered. Although the precise mechanism(s) by which exogenous NO inhibits ROCK activity is not known, it seems likely that it does so by inducing a conformational change. It is known that activated ROCKs reduce the stability of endothelial NO synthase mRNA and inhibit phosphatidylinositol 3-kinase/Akt signaling, leading to a decline in endogenous NO bioavailability. Exogenous NO may compensate for an imbalance of endogenous endothelial NO synthase/NO signaling in damaged VSMCs and/or endothelial cells. The question of whether exogenous NO affects the ROCK pathway directly or indirectly merits investigation. As the clinical use of selective ROCK inhibitors is presently extremely limited, it might also be of value to determine which of the currently available drugs most effectively and safely inhibit the ROCK pathways in the cardiovascular system.

To date, as clinicians, we have administered organic nitrates principally for the purpose of inducing vasodilation with consequent relief of chest symptoms. Although their clinical value in this respect will not change, at the same time a more pleiotropic effect, as in the case of the statins, may extend their clinical value in the near future.

1 Asanuma H, Sanada S, Asakura M, Asano Y, Kim J, Shinozaki Y, Mori H, Minamino T, Takashima S, Kitakaze M. Carperitide induces coronary vasodilation and limits infarct size in canine ischemic hearts: role of NO. Hypertens Res 2014; 37: 716-723.

2 Shimokawa H, Takeshita A. Rho-kinase is an important therapeutic target in cardiovascular medicine. Arterioscler Thromb Vasc Biol 2005; 25: 1767-1775.

3 Cao X, Luo T, Luo X, Tang Z. Resveratrol prevents AngIIinduced hypertension via AMPK activation and RhoA ROCK suppression in mice. Hypertens Res 2014; 37 803-810.

4 Riento K, Ridley AJ. Rocks: multifunctional kinases in cell behaviour. Nat Rev Mol Cell Biol 2003; 4: $446-456$

5 Sawada N, Liao JK. Rho/Rho-associated coiled-coil forming kinase pathway as therapeutic targets for statins in atherosclerosis. Antioxid Redox Signal 2014; 20: 1251-1267.

6 Kimura K, Ito M, Amano M, Chinara K, Fukata Y, Nakafuka M, Yamamori B, Feng J, Nakano T, Okawa K, Iwamatsu A, Kaibuchi K. Regulation of myosin phosphatase by Rho and Rho-associated kinase (Rho-kinase). Science 1996; 273: 245-248.

7 Sauzeau V, Le Jeune $H$, Cario-Toumaniantz C, Smolenski A, Lohmann SM, Bertoglio J, Chardin P, Pacaud P, Loirand G. Cyclic GMP-dependent protein kinase signaling pathway inhibits $\mathrm{RhoA}$-induced $\mathrm{Ca} 2+$ sensitization of contraction in vascular smooth muscle. J Biol Chem 2000; 275: 21722-21729.

8 Chitaley K, Webb RC. Nitric oxide induces dilation of rat aorta via inhibition of rho-kinase signaling. Hypertension 2002; 39: 438-442.

9 Mohri M, Shimokawa H, Hirakawa Y, Masumoto A, Takeshita A. Rho-kinase inhibition with intracoronary fasudil prevents myocardial ischemia in patients with coronary microvascular spasm. J Am Coll Cardiol 2003 41: 15-19.

10 Maruhashi T, Noma K, Fujimura N, Kajikawa M, Matsumoto T, Hidaka T, Nakashima A, Kihara Y, Liao JK, Higashi Y. Exogenous nitric oxide inhibits Rho-associated kinase activity in patients with angina pectoris: a randomized controlled trial. Hypertens Res 2015; 38: 485-490.

11 Maruhashi T, Noma K, Iwamoto Y, Iwamoto A, Oda N, Kajikawa $M$, Matsumoto $T$, Hidaka $T$, Kihara $Y$, Chayama K, Nakashima A, Goto C, Liao JK, Higashi $Y$. Critical role of exogenous nitric oxide in ROCK activity in vascular smooth muscle cells. PLoS One 2014; 9: e109017.

12 Hata T, Goto C, Soga J, Hidaka T, Fujii Y, Idei N, Fujimura N, Maruhashi T, Mikami S, Kihara $Y$, Chayama K, Noma K, Liao JK, Higashi Y. Measurement of Rho-associated kinase (ROCK) activity in humans: validity of leukocyte $\mathrm{p}-\mathrm{MBS} / \mathrm{t}-\mathrm{MBS}$ in comparison with vascular response to fasudil. Atherosclerosis 2011; 214: 117-121.

13 Kikuchi Y, Yasuda S, Aizawa K, Tsuburaya R, Ito Y, Takeda M, Nakayama M, Ito K, Takahashi J, Shimokawa H. Enhanced Rho-kinase activity in circulating neutrophils of patients with vasospastic angina: a possible biomarker for diagnosis and disease activity assessment. J Am Coll Cardiol 2011; 58: 1231-1237. 